\title{
EFFECT OF GROWTH TEMPERATURE ON THE FATTY ACID COMPOSITION OF A BLUE-GREEN ALGA*
}

\author{
Raymond W. Holton, Harry H. Blecker, and Michael Onore \\ Department of Botany, University of Tennessee, Knoxville, \\ and Flint College, University of Michigan, Flint
}

(Received 11 March 1964)

\begin{abstract}
The fatty acid composition of the blue-green alga, Anacystls nidulans, was investigated by gasliquid chromatography at four different growth temperatures with illumination, aeration, cell density, medium composition, and growth rate kept constant. At all temperatures palmitic acid and a hexadecenoic acid presumed to be paimitoleic totaled approximately $90 \%$ of the fatty acids present but the ratio of the hexedecenoic to palmitic decreased as the temperature was raised. An octadecenoic and tetradecenoic (probably oleic and myristoleic, respectively) were also present and traces of a heptadecenoic acid and of others were detected. At $26^{\circ}, 32^{\circ}$, and $35^{\circ}$, the ratio of total unsaturated to saturated acids remained approximately 1.0 although the qualitative composition changed, but at $41^{\circ}$ the saturated acids predominated, the ratio being 0.7 . In contrast to other algae and higher plants, polyunsaturated acids were absent in Anacystis which in this way resembles the photosynthetic bacteria.
\end{abstract}

AN INVESTIGATION of the fatty acid composition of a blue-green alga is of interest from several points of view. First, a complete fatty acid analysis of a blue-green alga is not available. Mazur and Clarke ${ }^{1}$ found that saturated acids (free and combined) made up 20.6\% and unsaturated acids $30.9 \%$ of the total lipid of Gloeotrichia obtained from a water bloom. More recently, Scheuerbrandt and Bloch ${ }^{2}$ reported the presence of palmitoleic as $27 \%$ and oleic as $12 \%$ of the total fatty acids in Anabaena variabilis from a laboratory culture and referred to unpublished data on the presence of polyunsaturated acids in that organism. ${ }^{3}$ In spite of the interesting and apparently primitive phylogenetic position of the Cyanophyceae, no further data appear to be available on their fatty acid composition.

A second point of interest is that the blue-green algae, along with the bacteria, are the only groups of organisms which contain truly thermophilic species. The nature of the mechanism by which thermophilic organisms are able to withstand temperatures that are fatal to mesophilic organisms has long intrigued biologists. Experimental data supporting a number of theories have been advanced 4,5 including the suggestion that the degree of saturation of the lipids is related to the minimum growth temperature. ${ }^{6}$ Thus at higher growth temperatures, the lipids of an organism would be expected to be more saturated than when the same organism is grown at a lower temperature. Conversely, it was suggested that the minimum temperature for growth is similar to that at which the lipids solidify. ${ }^{6}$ Some data are available on the

* Contribution from the Botanical Laboratory, The University of Tennessee, Knoxville, Tennessee, U.S.A., N. Ser. 247.

1 A. Mazur and H. T. Clarke, J. Biol. Chem. 143, 39 (1942).

2 G. SCheuerarandt and K. Bloch, J. Bial. Chem. 237, 2064 (1962).

3 J. ERWn and K. BLoch, Biochem. Z. 338, 496 (1963).

4 H. KoffLER, Bacteriol. Rev. 21, 227 (1957).

5 M. B. Allen, in Comparative Biochemistry, Vol. 1, p. 487, Academic Press, New York (1960).

6 E. R. L. Gaughran, J. Bacteriol. 53, 506 (1947).

40 
fatty acid composition of lipids of thermophilic bacteria and the effect of temperature on bacterial lipids and it would be of interest to have similar information on the blue-green algae.

Cultural and environmental conditions other than temperature are known to affect the lipid constitution of algae. ${ }^{7}$ The green alga, Chlorella, has been extensively studied ${ }^{8,9}$ and the lipid content was shown to be affected by light, $\mathrm{CO}_{2}$ concentration, and nitrogen source in the growth medium. In the blue-green algae, Collyer and $\mathrm{Fogg}^{10}$ found that a deficiency in available water as seen in growth on agar medium vs. liquid culture increased the total amount of lipids present in Anabaena and Oscillatoria. However, nitrogen deficiency did not increase the lipid content as in Chlorella. In order to ascribe changes in fatty acid composition to effects of temperature alone, it is therefore necessary to hold the other environmental and growth variables constant.

We report below the use of gas-liquid chromatography to study the fatty acid composition of the blue-green alga, Anacystis nidulans, grown under highly controlled conditions.

\section{Algal Growth Characteristics}

\section{RESULTS}

The known effects of environment and growth conditions on both the qualitative and quantitative lipid constitution prescribe completely controlled growth conditions. The nature of the steady-state culture chamber ${ }^{11}$ insures that the illumination, cell density, growth rate, temperature, aeration, and nutrient composition remain constant. To insure that constant

Table 1. Growth characteristics of algae grown UNDER STANDARD CONDITIONS AT VARIOUS TEMPERATURES

\begin{tabular}{ccc}
$\begin{array}{c}\text { Temp. } \\
\left({ }^{\circ} \mathrm{C}\right)\end{array}$ & $\begin{array}{c}\text { Specific growth } \\
\text { rate* } \\
\left(\log _{10} \text { units/day) }\right.\end{array}$ & $\begin{array}{c}\text { Average dry weight } \dagger \\
(\mathrm{mg} / \mathrm{ml})\end{array}$ \\
\hline 26.0 & $0.48(20)$ & $0.94 \pm 0.01(6)$ \\
32.2 & $0.39(35)$ & $1.20 \pm 0.24(10)$ \\
41.2 & $0.49(44)$ & $0.90 \pm 0.04(8)$ \\
\hline
\end{tabular}

* The figure in parentheses is the number of volume measurements made and used in the calculation of the growth rate.

$\uparrow$ The range is the standard deviation from the mean and the figure in parentheses is the number of dry weight determinations.

growth conditions were maintained in successive growth chambers at the same temperature. data used in the calculation of cell density and growth rate were obtained for each successive chamber and are summarized in Table 1.

While the growth rates were very similar at $26^{\circ}$ and $41^{\circ}$, the rate at $32^{\circ}$ was slightly less as would be expected from the somewhat higher cell concentration maintained. (The relatively large standard deviation in dry weights at $32^{\circ}$ was due to fluctuation in cell density in successive

7 J. D. A. MrLter, in Physiology and Biochemistry of Algae, p. 357, Academic Press, New York (1962).

${ }^{8}$ H. A. Spoehr and H. W. Mriner, Plant Physiol. 24, 120 (1949).

9 H. W. Milner, in Algal Culture-From Laboratory to Pilot Plant, p. 285, Carnegie Institution of Washington Publication 600, Washington, D.C. (1953).

10 D. M. Collyer and G. E. FooO, J. Exp. Botany 6, 256 (1955).

11 J. MYers and L. B. Crark, J. Gen. Physiol. 28, 103 (1944). 
chambers rather than to variations in duplicate analyses of a single chamber.) At all temperatures the growth rates were less than maximum ${ }^{12,13}$ and were limited by effective light intensity so that they are little affected by temperature. Practically speaking, the environmental and growth conditions were quite uniform and it is assumed that differences found in the fatty acid composition were due to temperature effects alone.

\section{Identification of Fatty Acid Esters}

From gas-liquid chromatograms such as those in Fig. 1, the retention times for the esters present were obtained and the esters identified from a plot of the $\log _{10}$ retention time vs.

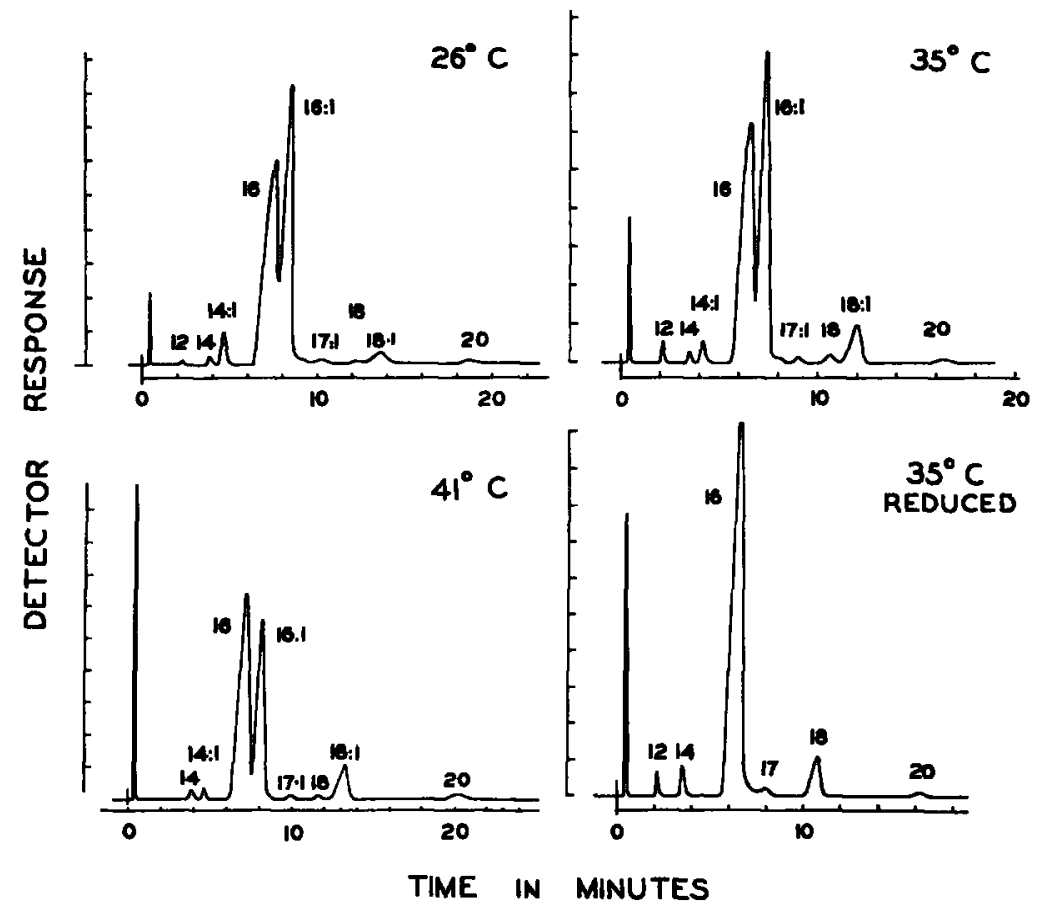

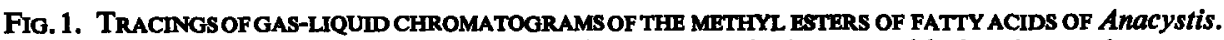
Lower right, cells grown at $35^{\circ}$ but the fatty acid sample was hydrogenated before it was chromatographed. The sample sizes were $10 \mu \mathrm{l}$ at $41^{\circ}, 20 \mu \mathrm{l}$ at $26^{\circ}$, and $15 \mu \mathrm{l}$ at $35^{\circ}$; other conditions are given in the text. The first peak (from the left) in each case is the solvent, hexane, and the other peaks are identified by carbon chain length and number of double bonds (see Table 2). The abscissa is the elapsed time after injection of the sample.

number of carbon atoms of known esters. Esters of palmitic and a hexadecenoic acid were the principal ones present with lesser amounts of those from an octadecenoic, a tetradecenoic, stearic, myristic, arachidic, a heptadecenoic acid, and traces of lauric acid also being detected.

That the presumed mono-unsaturated acids were such was confirmed by hydrogenation of an ester mixture which gave the expected saturated esters (Fig. 1 lower right) with the calculated uptake of hydrogen. No expected peaks were observed. The retention time data on the usual polyester column and on an Apiezon L column are consistent with the interpretation that the mono-unsaturated acids detected are the common $\Delta-9$ isomers, that is,

12 J. Myers and W. A. Kratz, J. Gen. Physiol. 39, 11 (1955).

13 W. A. Kratz and J. MYers, Amer. J. Botany 42, 282 (1955). 
palmitoleic, oleic, and myristoleic acids. It is recognized that unequivocal localization of the double bond requires chemical degradation of the acids, which when done with Anabaena mono-unsaturated acids by Scheuerbrandt and Bloch ${ }^{2}$ established the presence of palmitoleic and oleic acids in that blue-green alga.

The only uncommon acid found was a $\mathrm{C}_{17}$ acid present in small amounts. From retention time data and the hydrogenation experiment, it was identified as the singly unsaturated $\mathrm{C}_{17}$ acid. The retention time data agree with those for the $\mathrm{C}_{17}$ acids in butter fat. ${ }^{14}$ That it was not a branched chain acid was shown by running the ester sample at different temperatures and noting that the change in retention time was that expected for unsaturated rather than branched chain acids. While the occurrence of "odd-number" carbon acids is relatively rare in nature, $\mathrm{C}^{17}$ acids have been also reported in the green alga, Chlorella, ${ }^{15}$ the phytoflagellate, Euglena, ${ }^{16}$ in certain yeasts ${ }^{17}$ as well as in butter-fat. ${ }^{14}$

\section{Effect of Growth Temperature on the Fatty Acid Composition}

Fatty acid ester analyses of Anacystis grown at four different temperatures are summarized in Table 2. The dominant acids are the $\mathrm{C}_{16}$ acids, palmitic and the hexadecenoic, which

TABLE 2. EFFECT OF TEMPERATURE ON THE FATTY ACID COMPOSITION ( $\%$ BY WEIGHT OF TOTAL) OF Anacystis

\begin{tabular}{|c|c|c|c|c|c|}
\hline \multirow{2}{*}{\multicolumn{2}{|c|}{$\begin{array}{c}\text { Fatty acid } \\
\text { (C atoms: double bonds) }\end{array}$}} & \multicolumn{4}{|c|}{ Growth temperature $\left({ }^{\circ} \mathrm{C}\right)$} \\
\hline & & \multirow{2}{*}{$\frac{26.0^{*}}{\text { trace } f}$} & \multirow{2}{*}{$\frac{32 \cdot 2}{\text { trace }}$} & \multirow{2}{*}{$\frac{35.0 \dagger}{\text { trace }}$} & \multirow[t]{2}{*}{$41 \cdot 2$} \\
\hline Lauric & $12: 0$ & & & & \\
\hline Myristic & $14: 0$ & 0.9 & 0.5 & 0.6 & 0.8 \\
\hline Tetradecenoic & $14: 1$ & $3 \cdot 3$ & 1.0 & $1 \cdot 3$ & 0.7 \\
\hline Palmitic & $16: 0$ & $47 \cdot 3$ & 47.0 & $47 \cdot 3$ & 57.6 \\
\hline Hexadecenoic & $16: 1$ & $44 \cdot 1$ & $38 \cdot 8$ & 40.2 & $31 \cdot 3$ \\
\hline Heptadecenoic & $17: 1$ & 0.6 & 0.5 & 0.8 & 0.5 \\
\hline Stearic & $18: 0$ & 0.6 & 1.4 & 0.8 & 0.5 \\
\hline Octadecenoic & $18: 1$ & $2 \cdot 7$ & 10.0 & 8.5 & $8 \cdot 0$ \\
\hline Arachidic & $20: 0$ & 0.5 & 0.7 & 0.7 & 0.6 \\
\hline \multicolumn{2}{|c|}{ Hexadecenoic/palmitic } & 0.93 & 0.83 & 0.85 & 0.54 \\
\hline \multicolumn{2}{|c|}{ Unsaturated/saturated } & 1.01 & 1.02 & 1.02 & 0.68 \\
\hline \multicolumn{2}{|c|}{ Average $C$ chain length } & $15 \cdot 9$ & $16 \cdot 2$ & $16 \cdot 3$ & $16 \cdot 2$ \\
\hline
\end{tabular}

* In this experiment, the extracted lipids totaled $11 \%$ of the dry weight of the algae.

$\dagger$ Algae grown at this temperature were cultured under "non-standard" conditions of illumination and growth rate (see Experimental).

¥ "Trace" indicates a definite peak, but one that is less than $0-5 \%$ of the total.

account for between 86 and $91 \%$ by weight of the fatty acid esters at all temperatures studied. Palmitic remains constant at the low temperatures but increases dramatically at $41^{\circ}$. The hexadecenoic, on the other hand, decreases as the temperature increases. While tetradecenoic and octadecenoic also appear to show composition trends with temperature, they were present in so much lower concentration that the differences found may not be significant. Possibly of greater significance is the ratio of the total unsaturated to saturated acids which remains 1.0

14 R. P. Hansen, F. B. Shorland and J. J. Cooke, New Zealand J. Sci. Technol. 6, 101 (1963).

15 H. Schlenk, H. K. Mangold, J. L. Gellerman, W. E. Link, R. A. Morrissette, R. T. Holman and H. HAYES, J. Am. Oil Chemists' Soc. 37, 547 (1960)

16 E. D. KorN, Biochem. Biophys. Res. Comm. 14, 1 (1964).

17 M. Kates and R. M. BaXter, Can. J. Biochem. Physiol. 40, 1213 (1962). 
at temperatures of $26^{\circ}$ to $35^{\circ}$ and decreases only at the $41^{\circ}$ culture condition. The hexadecenoic/ palmitic ratio, on the other hand, shows a decrease with increasing temperature. While $26^{\circ}$ to $35^{\circ}$ temperature has little effect on unsaturation, growth at $26^{\circ}$ does result in acids of slightly shorter average carbon chain length than at $32^{\circ}$ and above.

\section{DISCUSSION}

The data presented here are the first complete analyses of the fatty acid composition of a blue-green alga. The most striking observations are the lack of "unusual" fatty acids often found in bacteria ${ }^{18}$ with which the blue-green algae bear cytological resemblances, and the lack of the highly unsaturated lipids found in other algae $3,5,19-21$ and in higher plants ${ }^{22-24}$ with which the Cyanophyceae share physiological similarities. If simplicity in fatty acid composition has any direct relationship to biochemical evolution in the plant kingdom, certainly these data reaffirm the blue-green algae's supposed primitive phylogenetic position.

Recently, Erwin and Bloch ${ }^{3}$ found high concentrations of $\alpha$-linolenic acid in the green flagellates, Euglena and Chlamydomonas, when grown photosynthetically, but much lower concentrations of this acid when they were grown heterotrophically. In higher plants, linolenic acid is present in photosynthetic tissues such as the leaves but absent in stems; however, in photosynthetic bacteria, polyunsaturated acids are absent. ${ }^{2,25}$ Erwin and Bloch ${ }^{3}$ concluded that $\alpha$-linolenic acid is a characteristic constituent of those organisms in which photosynthesis is of the green plant type. They include the blue-green algae in this group because $\alpha$-linolenic acid was found in Anabaena, the only blue-green alga thus far examined in that laboratory (Bloch, personal communication). Their conclusion from comparative data on composition and the effects of various photosynthetic inhibitors on acetate incorporation into the unsaturated fatty acids of Euglena suggested that linolenic acid is involved either chemically or physically in the reactions leading to oxygen evolution. On the other hand, our data on the only blue-green that we have studied show that Anacystis is similar to the photosynthetic bacteria in lacking $\alpha$-linolenic and other polyunsaturated acids. Our data infer that these acids might be part of the chloroplast membrane which is lacking in bacteria and blue-green algae and that the polyunsaturated acids are of structural importance rather than being related to a specific biochemical reaction. The variation in fatty acid content with environmental conditions discussed below would seem to strengthen our conclusion, but more data are needed before any theory can be strongly advocated.

Recently, the effect of growth temperature on the fatty acid composition of bacteria 26,27 and of several yeasts ${ }^{17}$ has been investigated in detail by means of GLC. (These references, particularly the latter one, include detailed reviews of the earlier literature on the effects of growth temperature.) With Escherichia coli grown at eight temperatures from $10^{\circ}$ to $43^{\circ}$, there was a steady increase of saturated acids and a decrease of unsaturated acids with increasing temperature. ${ }^{26}$ With Serratia marsecens ${ }^{27}$ the amount of unsaturated acids

18 W. M. O'LeARY, Bacteriol. Rev. 26, 421 (1962).

19 M.-H. LaUR, Compt. rend. 253, 966 (1961).

20 V. R. WilliaMs and R. MCMIILAN, Science 133, 456 (1961).

21 E. KLenK, W. KNIPPRath, D. EberhaGen and H. P. Koof, Z. physiol. Chem. 334, 44 (1963).

22 F. T. Wolf, J. G. Coniguo and J. T. DAvis, Plant Physiol. 37, 83 (1962).

23 H. Debuch, $Z$. Naturforsch. 16b, 561 (1961).

24 F. B. Shorl.AND, in Comparative Biochemistry, Vol. 3, p. 1, Academic Press, New York (1962).

25 A. R. HANDS and W. BARTLEY, Biochem. J. 84, 238 (1962).

26 A. G. MARR and J. L. INGRAHAM, J. Bacterial. 84, 1260 (1962).

${ }^{27}$ D. G. BisHOP and J. L. STiLt, J. Lipid Res. 4, 87 (1963). 
remained about $10 \%$ of the total at $30^{\circ}$ and $37^{\circ}$ but at $20^{\circ}$ approached $50 \%$. The data on yeasts (Candida spp.) ${ }^{17}$ were obtained at only two temperatures but the expected tendency towards greater proportion of more saturated acids at higher temperatures was again seen. Marr and Ingraham ${ }^{26}$ concluded that the fatty acid composition is not directly related to the temperature limits of growth since changes of media composition also resulted in major changes in fatty acid composition.

The data on $E$. coli are of particular interest when compared to our data on Anacystis since, in these studies, the effects of other environmental and growth variables were rigidly controlled and the analyses were made at more than two temperatures. With $E$. coli, there is a continual shift towards saturation with increasing temperature in contrast to Anacystis in which a definite ratio of saturation to unsaturation is found in the $26^{\circ}$ to $35^{\circ}$ temperature range and increased saturation occurs only at $41^{\circ}$. Our data suggest that in Anacystis there is a temperature minimum for saturation and unsaturation levels. However, this minimum saturation/unsaturation level does not set a temperature limit for growth which can occur at lower temperatures at the same rate as at temperatures where the saturation/unsaturation level is higher. While Anacystis cannot be considered a truly thermophilic alga, its upper temperature range for growth is greater than that of many blue-green algae and the ease with which it is grown under constant environmental conditions made it the organism of choice for these experiments.

No consistent pattern emerges for the effect of temperature on fatty acid composition as related to growth with the limited data available on bacteria and blue-green algae. In fact, the conclusion of Marr and Ingraham ${ }^{26}$ about $E$. coli is probably also applicable to Anacystis; namely, that rather significant changes in fatty acid composition bear little direct relationship to the growth rate and that such differences in composition have only trivial effects on the physiology of the organism.

\section{Growth of the Alga}

\section{EXPERIMENTAL}

Anacystis nidulans Drouet was grown under steady state conditions in an all-glass automatic culture apparatus whose design was patterned after a Liebig condenser. ${ }^{11}$ Medium $C$ of $\mathrm{Kratz}$ and $\mathrm{Myers}{ }^{13}$ was the nutrient source. The algal culture was aerated with $0.5 \% \mathrm{CO}_{2}$ in air and illuminated with ten $25 \mathrm{~W}$ incandescent lamps. For reasons given earlier ${ }^{12}$ no attempt was made to measure light intensity. A full culture chamber had a volume of about $350 \mathrm{ml}$ of which $300 \mathrm{ml}$ were removed approximately every 2 days leaving $50 \mathrm{ml}$ for an inoculum. The algae were harvested by centrifugation at $1700 \mathrm{~g}$ for $10 \mathrm{~min}$ and were washed once with distilled water. Duplicate 5-ml aliquots of the harvest were treated similarly and dried in aluminum pans at $105^{\circ}$ to determine the dry weight of algae present per ml of harvest. The algae were then lyophilized and stored in the dark in a desiccator with a nitrogen atmosphere at $-10^{\circ}$. When it was necessary to send the algae through the mail, they were sent in lyophilized form in a vial with a nitrogen atmosphere and on arrival were placed in a deep freeze and kept at $-40^{\circ}$.

Growth in the continuous culture apparatus is logarithmic and is defined by the growth equation, $\log _{10} N / N_{0}=k t$ where $N_{0}$ is volume in $\mathrm{ml}$ of inoculum at the beginning of a growth period, $N$ is the total $\mathrm{ml}$ of culture at the end of a growth period, $t$ is the time in days, and $k$ is the specific growth rate. ${ }^{28}$ In these experiments, the chamber was calibrated by volume before

28 J. MYers, J. Gen. Physiol. 29, 419 (1946). 
inoculation so that the increases in volume of the algal culture could be recorded over different time intervals during the experiment. For convenience, $k$ is defined in terms of $\log _{10}$ units per day and evaluated from a plot of $\log _{10} N / N_{0}$ vs. $t$. Thus, a $k$ of 0.301 indicates a generation time of 1 day.

Besides the algae grown under the standard conditions described above, algae were also obtained from a larger culture apparatus kept at $35^{\circ}$ which was under manual control of a constant daily harvest and dilution. Under these conditions, both growth rate and illumination intensity varied during the growth period but the yields from this chamber were much greater and therefore used for analyses at $35^{\circ}$ as is noted in Table 2.

\section{Extraction of the Lipids}

Approximately $1 \mathrm{~g}$ or more of lyophilized cells was refluxed with $95 \%$ methanol (1:15 by weight) for $1 \mathrm{hr}$; the residue was removed by filtration and similarly re-extracted. The residue from the second extraction was extracted twice with absolute methanol ( $1: 15$ by weight) for $1 \mathrm{hr}$ each time. The combined methanol filtrates were centrifuged to clarify them, if necessary, and the solvent was removed under reduced pressure in a nitrogen atmosphere. The resulting residue was taken up in dry, peroxide-free ether and any insoluble non-lipid materials were removed by centrifugation. The insoluble material was washed once with ether.

The algal residue from the methanol extractions was then refluxed with peroxide-free ether ( $1: 15$ by weight) for $1 \mathrm{hr}$, filtered, and the resultant residue re-extracted twice in the same manner.

All of the ether solutions were combined and the solvent removed under reduced pressure in a nitrogen atmosphere leaving the extracted lipid material. The final insoluble algal residue was a bluish-white, free-flowing powder.

\section{Saponification and Esterification Procedures}

The extracted lipid material was refluxed in a 10-fold excess of $5 \%$ methanolic KOH for $4 \mathrm{hr}$. After removal of about one-half of the methanol by distillation under reduced pressure in a nitrogen atmosphere, an equal volume of water was added and the resulting solution was extracted three times with peroxide-free ether to remove non-saponifiable material. The aqueous layer was separated, acidified with $6 \mathrm{~N}$ sulfuric acid, and then extracted four times with pure heptane to remove the free fatty acids.

After evaporation of the heptane under reduced pressure in a nitrogen atmosphere, the acids were esterified with diazomethane. ${ }^{29}$ (Best results were obtained with a flow rate of 0.2 $\mathrm{ml} \mathrm{N}_{2} / \mathrm{min}$.) Analysis of the resultant etheral solution of the esters showed that methyl laurate was the lowest molecular weight ester present and so usually the ether was removed under reduced pressure in a nitrogen atmosphere and the ester mixture taken up in pure heptane. This mixture was stored under nitrogen at $-40^{\circ}$ until used.

In the experiments where a portion of the esters were reduced before analyses, a platinum oxide catalyst reduction procedure ${ }^{30}$ was used without modification.

29 H. Schilenk and J. L. Gellerman, Anal. Chem. 32, 1412 (1960).

30 J. W. Farquhar, W. Insull, JR., P. Rosen, W. Stoffel and E. H. Ahrens, JR., Nutrition Rev. Suppl. 17 (8), Part 2, 1 (1959). 


\section{Gas Chromatographic Analyses}

All the analyses were carried out using a Perkin-Elmer Model 154-D Vapor Fractometer with a $2-\mathrm{m}$ diethylene glycol succinate polyester column at $205^{\circ}$ with a flow rate of $80 \mathrm{ml}$ helium/min. The detector was a thermal conductivity cell fitted with thermistors. Known esters obtained from commercial sources were used as standards.

In order to detect and measure minor constituents as well as to obtain accurate retention times for the major components, three concentrations of each ester mixture were run. Dilute mixtures were used to establish the retention times of the major components and intermediate and concentrated mixtures were used to detect minor constituents. Concentrations were determined by peak area measurements with internal normalization. Comparisons of the calculated concentration obtained from different extractions of the same batch of algae agreed very well as did comparisons between intermediate and concentrated samples of the same batch.

To insure the accuracy in the identification of the esters was not being influenced by transesterification which is said to occur at high temperatures, the following experiment was done. A mixture of known saturated esters $\left(C_{14}\right.$ through $\left.C_{20}\right)$ and unsaturated esters (palmitoleic through linolenic) were run at the usual $205^{\circ}$ and also at $180^{\circ}$ where transesterification is reported to be unimportant. ${ }^{31}$ The results did not show any substantial or regular changes with either the saturated or unsaturated esters. The lesser importance of transesterification in our experiments may be due to our use of a low column loading and hence relatively short retention times. The higher temperature was used routinely because the retention times at $180^{\circ}$ were too long even at flow rates of $180 \mathrm{ml} / \mathrm{min}$. Higher flow rates would have required too great a $P_{i} / P_{o}$ ratio.

It was noted early in the study that the retention times of the known standard esters decreased in a slow, regular way, probably due to a slow loss of column partition liquid. For this reason, a mixture of known saturated and unsaturated esters was run following every unknown ester mixture so that the identification could be made under identical conditions. Plots of the $\log _{10}$ apparent retention time (air peak to peak maximum) against carbon number were constructed for each homologous series for each analysis and the identification of the unknowns made from these plots.

Acknowledgements-The authors are grateful for a grant from the Rackham School of Graduate Studies, The University of Michigan, which made this study possible. The first author also acknowledges, with thanks, the use of the laboratory facilities of Professor Jack Myers, University of Texas, for the growth of the algae.

31 A. T. JAMEs, Methods Biochem. Anal. 8, 1 (1960). 